\title{
On Inverse Hilbert-Type Inequalities
}

\author{
Zhao Changjian ${ }^{1}$ and Wing-Sum Cheung ${ }^{2}$ \\ ${ }^{1}$ Department of Information and Mathematics Sciences, College of Science, China Jiliang University, \\ Hangzhou 310018, China \\ ${ }^{2}$ Department of Mathematics, The University of Hong Kong, Pokfulam Road, Hong Kong
}

Correspondence should be addressed to Zhao Changjian, chjzhao@163.com

Received 14 November 2007; Revised 1 December 2007; Accepted 4 December 2007

Recommended by Martin J. Bohner

This paper deals with new inverse-type Hilbert inequalities. Our results in special cases yield some of the recent results and provide some new estimates on such types of inequalities.

Copyright (C) 2008 Z. Changjian and W.-S. Cheung. This is an open access article distributed under the Creative Commons Attribution License, which permits unrestricted use, distribution, and reproduction in any medium, provided the original work is properly cited.

\section{Introduction}

Considerable attention has been given to Hilbert inequalities and Hilbert-type inequalities and their various generalizations by several authors including Handley et al. [1], Minzhe and Bicheng [2], Minzhe [3], Hu [4], Jichang [5], Bicheng [6], and Zhao [7, 8]. In 1998, Pachpatte [9] gave some new integral inequalities similar to Hilbert inequality (see [10, page 226]). In 2000, Zhao and Debnath [11] established some inverse-type inequalities of the above integral inequalities. This paper deals with some new inverse-type Hilbert inequalities which provide some new estimates on such types of inequalities.

\section{Main results}

Theorem 2.1. Let $0<p_{i} \leq 1(i=1, \ldots, n)$ and $r \leq 0$. Let $\left\{a_{i, m_{i}}\right\}$ be $n$ positive sequences of real numbers defined for $m_{i}=1,2, \ldots, k_{i}$, where $k_{i}(i=1, \ldots, n)$ are natural numbers, define $A_{i, m_{i}}=$ $\sum_{s_{i}=1}^{m_{i}} a_{i, s_{i}}$, and define $A_{i, 0}=0$. Then for $p^{-1}+q^{-1}=1, p<0$ or $0<p<1$, one has

$$
\sum_{m_{1}=1}^{k_{1}} \cdots \sum_{m_{n}=1}^{k_{n}} \frac{\prod_{i=1}^{n} A_{i, m_{i}}^{p_{i}}}{\left((1 / n) \sum_{i=1}^{n} m_{i}^{r}\right)^{n /(p r)}} \geq \prod_{i=1}^{n} p_{i} k_{i}^{1 / p}\left(\sum_{m_{i}=1}^{k_{i}}\left(k_{i}-m_{i}+1\right)\left(a_{i, m_{i}} A_{i, m_{i}}^{p_{i}-1}\right)^{q}\right)^{1 / q} .
$$


Proof. By using the following inequality (see [10, page 39]):

$$
h_{i} a_{i, m_{i}}^{h_{i}-1}\left(a_{i, m_{i}}-b_{i, m_{i}}\right) \leq a_{i, m_{i}}^{h_{i}}-b_{i, m_{i}}^{h_{i}} \leq h_{i} b_{i, m_{i}}^{h_{i}-1}\left(a_{i, m_{i}}-b_{i, m_{i}}\right),
$$

where $a_{i, m_{i}}>0, b_{i, m_{i}}>0$, and $0 \leq h_{i} \leq 1(i=1,2, \ldots, n)$, we obtain that

$$
\begin{gathered}
A_{i, m_{i}+1}^{p_{i}}-A_{i, m_{i}}^{p_{i}} \geq p_{i} A_{i, m_{i}+1}^{p_{i}-1}\left(A_{i, m_{i}+1}-A_{i, m_{i}}\right)=p_{i} a_{i, m_{i}+1} A_{i, m_{i}+1}^{p_{i}-1} \\
\sum_{m_{i}=0}^{k_{i}-1} A_{i, m_{i}+1}^{p_{i}}-A_{i, m_{i}}^{p_{i}}=A_{i, k_{i}}^{p_{i}} \geq \sum_{m_{i}=0}^{k_{i}-1} p_{i} a_{i, m_{i}+1} A_{i, m_{i}+1}^{p_{i}-1}=p_{i} \sum_{m_{i}=1}^{k_{i}} a_{i, m_{i}} A_{i, m_{i}}^{p_{i}-1},
\end{gathered}
$$

thus

$$
A_{i, m_{i}}^{p_{i}} \geq p_{i} \sum_{s_{i}=1}^{m_{i}} a_{i, s_{i}} A_{i, s_{i}}^{p_{i}-1} .
$$

From inequality (2.4) and in view of the following mean inequality and inverse Hölder's inequality [10, page 24], we have

$$
\begin{gathered}
\prod_{i=1}^{n} m_{i}^{1 / n} \geq\left(\frac{1}{n} \sum_{i=1}^{n} m_{i}^{r}\right)^{1 / r}, \\
\frac{\prod_{i=1}^{n} A_{i, m_{i}}^{p_{i}}}{\left((1 / n) \sum_{i=1}^{n} m_{i}^{r}\right)^{n /(p r)}} \geq \prod_{i=1}^{n} p_{i}\left(\sum_{s_{i}=1}^{m_{i}}\left(a_{i, s_{i}} A_{i, s_{i}}^{p_{i-1}}\right)^{q}\right)^{1 / q} .
\end{gathered}
$$

Taking the sum of both sides of $(2.6)$ over $m_{i}$ from 1 to $k_{i}(1,2, \ldots, n)$ first and then using again inverse Hölder's inequality, we obtain that

$$
\begin{aligned}
\sum_{m_{1}=1}^{k_{1}} \cdots \sum_{m_{n}=1}^{k_{n}} \frac{\prod_{i=1}^{n} A_{i, m_{i}}^{p_{i}}}{\left((1 / n) \sum_{i=1}^{n} m_{i}^{r}\right)^{n /(p r)}} & \geq \prod_{i=1}^{n} p_{i}\left(\sum_{m_{i}=1}^{k_{i}}\left(\sum_{s_{i}=1}^{m_{i}}\left(a_{i, s_{i}} A_{i, s_{i}}^{p_{i}-1}\right)^{q}\right)^{1 / q}\right) \\
& \geq \prod_{i=1}^{n} p_{i} k_{i}^{1 / p}\left(\sum_{m_{i}=1}^{k_{i}} \sum_{s_{i}=1}^{m_{i}}\left(a_{i, s_{i}} A_{i, s_{i}}^{p_{i}-1}\right)^{q}\right)^{1 / q} \\
& =\prod_{i=1}^{n} p_{i} k_{i}^{1 / p}\left(\sum_{s_{i}=1}^{k_{i}}\left(k_{i}-s_{i}+1\right)\left(a_{i, s_{i}} A_{i, s_{i}}^{p_{i}-1}\right)^{q}\right)^{1 / q} \\
& =\prod_{i=1}^{n} p_{i} k_{i}^{1 / p}\left(\sum_{m_{i}=1}^{k_{i}}\left(k_{i}-m_{i}+1\right)\left(a_{i, m_{i}} A_{i, m_{i}}^{p_{i}-1}\right)^{q}\right)^{1 / q}
\end{aligned}
$$

This completes the proof.

Remark 2.2. Taking $n=2, q=-2, r=-1$ to (2.1), (2.1) becomes

$$
\begin{aligned}
\sum_{m_{1}=1}^{k_{1}} \sum_{m_{2}=1}^{k_{2}} \frac{A_{1, m_{1}}^{p_{1}} A_{2, m_{2}}^{p_{2}}}{\left(m_{1}^{-1}+m_{2}^{-1}\right)^{-3}} & 8 p_{1} p_{2}\left(k_{1} k_{2}\right)^{3 / 2}\left(\sum_{m_{1}=1}^{k_{1}}\left(k_{1}-m_{1}+1\right)\left(a_{1, m_{1}} A_{1, m_{1}}^{p_{1}-1}\right)^{-2}\right)^{-1 / 2} \\
& \times\left(\sum_{m_{2}=1}^{k_{2}}\left(k_{2}-m_{2}+1\right)\left(a_{2, m_{2}} A_{2, m_{2}}^{p_{2}-1}\right)^{-2}\right)^{-1 / 2}
\end{aligned}
$$


Z. Changjian and W.-S. Cheung [9]:

This is just an inverse form of the following inequality which was proven by Pachpatte

$$
\sum_{m=1}^{k} \sum_{n=1}^{r} \frac{A_{m}^{p} B_{n}^{q}}{m+n} \leq \frac{1}{2} p q(k r)^{1 / 2}\left(\sum_{m=1}^{k}(k-m+1)\left(a_{m} A_{m}^{p-1}\right)^{2}\right)^{1 / 2}\left(\sum_{n=1}^{r}(r-n+1)\left(b_{n} B_{n}^{q-1}\right)^{2}\right)^{1 / 2} .
$$

Theorem 2.3. Let $\left\{a_{i, m_{i}}\right\}, A_{i, m_{i}}, k_{i}, p$, and $q$ be as defined in Theorem 2.1. Let $\left\{p_{i, m_{i}}\right\}$ be $n$ positive sequences for $m_{i}=1,2, \ldots, k_{i}(i=1,2, \ldots, n)$. Set $P_{i, m_{i}}=\sum_{S_{i}=1}^{m_{i}} p_{i, s_{i}}(i=1,2, \ldots, n)$. Let $\phi_{i}(i=$ $1,2, \ldots, n)$ be $n$ real-valued nonnegative, concave, and supermultiplicative functions defined on $R_{+}=$ $[0,+\infty)$. Then,

$$
\sum_{m_{1}=1}^{k_{1}} \cdots \sum_{m_{n}=1}^{k_{n}} \frac{\prod_{i=1}^{n} \phi_{i}\left(A_{i, m_{i}}\right)}{\left((1 / n) \sum_{i=1}^{n} m_{i}^{r}\right)^{n /(p r)}} \geq M\left(k_{1}, k_{2}, \ldots, k_{n}\right) \prod_{i=1}^{n}\left(\sum_{m_{i}=1}^{k_{i}}\left(k_{i}-m_{i}+1\right)\left(p_{i, m_{i}} \phi_{i}\left(\frac{a_{i, m_{i}}}{p_{i, m_{i}}}\right)\right)^{q}\right)^{1 / q},
$$

where

$$
M\left(k_{1}, k_{2}, \ldots, k_{n}\right)=\prod_{i=1}^{n}\left(\sum_{m_{i}=1}^{k_{i}}\left(\frac{\phi_{i}\left(P_{i, m_{i}}\right)}{P_{i, m_{i}}}\right)^{p}\right)^{1 / p} .
$$

Proof. From the hypotheses and by Jensen's inequality, the means inequality, and inverse Hölder's inequality, we obtain that

$$
\begin{aligned}
\prod_{i=1}^{n} \phi_{i}\left(A_{i, m_{i}}\right) & =\prod_{i=1}^{n} \phi_{i}\left(\frac{P_{i, m_{i}} \sum_{S_{i}=1}^{m_{i}} p_{i, s_{i}}\left(a_{i, s_{i}} / p_{i, s_{i}}\right)}{\sum_{s_{i}=1}^{m_{i}} p_{i, S_{i}}}\right) \geq \prod_{i=1}^{n} \phi_{i}\left(P_{i, m_{i}}\right) \phi_{i}\left(\frac{\sum_{S_{i}=1}^{m_{i}} p_{i, s_{i}}\left(a_{i, S_{i}} / p_{i, s_{i}}\right)}{\sum_{S_{i}=1}^{m_{i}} p_{i, s_{i}}}\right) \\
& \geq \prod_{i=1}^{n} \frac{\phi_{i}\left(P_{i, m_{i}}\right)}{P_{i, m_{i}}} \sum_{s_{i}=1}^{m_{i}} p_{i, s_{i}} \phi_{i}\left(\frac{a_{i, S_{i}}}{p_{i, S_{i}}}\right) \geq \prod_{i=1}^{n} \frac{\phi_{i}\left(P_{i, m_{i}}\right)}{P_{i, m_{i}}} m_{i}^{1 / p}\left(\sum_{s_{i}=1}^{m_{i}}\left(p_{i, s_{i}} \phi_{i}\left(\frac{a_{i, s_{i}}}{p_{i, S_{i}}}\right)\right)^{q}\right)^{1 / q} \\
& \geq\left(\frac{1}{n} \sum_{i=1}^{n} m_{i}^{r}\right)^{n /(p r)} \prod_{i=1}^{n} \frac{\phi_{i}\left(P_{i, m_{i}}\right)}{P_{i, m_{i}}}\left(\sum_{s_{i}=1}^{m_{i}}\left(p_{i, s_{i}} \phi_{i}\left(\frac{a_{i, s_{i}}}{p_{i, s_{i}}}\right)\right)^{q}\right)^{1 / q} .
\end{aligned}
$$

Dividing both sides of $(2.12)$ by $\left((1 / n) \sum_{i=1}^{n} m_{i}^{r}\right)^{n /(p r)}$ and then taking the sum over $m_{i}(i=$ $1,2, \ldots, n)$ from 1 to $k_{i}$ (and in view of inverse Hölder's inequality), we have

$$
\begin{aligned}
\sum_{m_{1}=1}^{k_{1}} \ldots \sum_{m_{n}=1}^{k_{n}} \frac{\prod_{i=1}^{n} \phi_{i}\left(A_{i, m_{i}}\right)}{\left((1 / n) \sum_{i=1}^{n} m_{i}^{r}\right)^{n /(p r)}} & \geq \prod_{i=1}^{n}\left(\sum_{m_{i}=1}^{k_{i}} \frac{\phi_{i}\left(P_{i, m_{i}}\right)}{P_{i, m_{i}}}\left(\sum_{s_{i}=1}^{m_{i}}\left(p_{i, s_{i}} \phi_{i}\left(\frac{a_{i, s_{i}}}{p_{i, s_{i}}}\right)\right)^{q}\right)^{1 / q}\right) \\
& \geq \prod_{i=1}^{n}\left(\sum_{m_{i}=1}^{k_{i}}\left(\frac{\phi_{i}\left(P_{i, m_{i}}\right)}{P_{i, m_{i}}}\right)^{p}\right)^{1 / p}\left(\sum_{m_{i}=1}^{k_{i}} \sum_{s_{i}=1}^{m_{i}}\left(p_{i, s_{i}} \phi_{i}\left(\frac{a_{i, s_{i}}}{p_{i, s_{i}}}\right)\right)^{q}\right)^{1 / q} \\
& =M\left(k_{1}, k_{2}, \ldots, k_{n}\right) \prod_{i=1}^{n}\left(\sum_{m_{i}=1}^{k_{i}} \sum_{s_{i}=1}^{m_{i}}\left(p_{i, s_{i}} \phi_{i}\left(\frac{a_{i, s_{i}}}{p_{i, s_{i}}}\right)\right)^{q}\right)^{1 / q} \\
& =M\left(k_{1}, k_{2}, \ldots, k_{n}\right) \prod_{i=1}^{n}\left(\sum_{m_{i}=1}^{k_{i}}\left(k_{i}-m_{i}+1\right)\left(p_{i, m_{i}} \phi_{i}\left(\frac{a_{i, m_{i}}}{p_{i, m_{i}}}\right)\right)^{q}\right)^{1 / q} .
\end{aligned}
$$

The proof is complete. 
Remark 2.4. Taking $n=2, q=-2, r=-1$ to (2.10), (2.10) becomes

$$
\begin{aligned}
\sum_{m_{1}=1}^{k_{1}} \sum_{m_{2}=1}^{k_{2}} \frac{\phi_{1}\left(A_{1, m_{1}}\right) \phi_{2}\left(A_{2, m_{2}}\right)}{\left(m_{1}^{-1}+m_{2}^{-1}\right)^{-3}} \geq & M\left(k_{1}, k_{2}\right)\left(\sum_{m_{1}=1}^{k_{1}}\left(k_{1}-m_{1}+1\right)\left(p_{1, m_{1}} \phi_{1}\left(\frac{a_{1, m_{1}}}{p_{1, m_{1}}}\right)\right)^{-2}\right)^{-1 / 2} \\
& \times\left(\sum_{m_{2}=1}^{k_{2}}\left(k_{2}-m_{2}+1\right)\left(p_{2, m_{2}} \phi_{2}\left(\frac{a_{2, m_{2}}}{p_{2, m_{2}}}\right)\right)^{-2}\right)^{-1 / 2},
\end{aligned}
$$

where

$$
M\left(k_{1}, k_{2}\right)=8\left(\sum_{m_{1}=1}^{k_{1}}\left(\frac{\phi_{1}\left(P_{1, m_{1}}\right)}{P_{1, m_{1}}}\right)^{2 / 3}\right)^{3 / 2}\left(\sum_{m_{2}=1}^{k_{2}}\left(\frac{\phi_{2}\left(P_{2, m_{2}}\right)}{P_{2, m_{2}}}\right)^{2 / 3}\right)^{3 / 2} .
$$

This is just an inverse of the following inequality which was proven by Pachpatte [9]:

$$
\begin{aligned}
\sum_{m=1}^{k} \sum_{n=1}^{r} \frac{\phi\left(A_{m}\right) \psi\left(B_{n}\right)}{m+n} \leq & M(k, r)\left(\sum_{m=1}^{k}(k-m+1)\left(p_{m} \phi\left(\frac{a_{m}}{p_{m}}\right)\right)^{2}\right)^{1 / 2} \\
& \times\left(\sum_{n=1}^{r}(r-n+1)\left(q_{n} \psi\left(\frac{b_{n}}{q_{n}}\right)\right)^{2}\right)^{1 / 2}
\end{aligned}
$$

where

$$
M(k, r)=\frac{1}{2}\left(\sum_{m=1}^{k}\left(\frac{\phi\left(P_{m}\right)}{P_{m}}\right)^{2}\right)^{1 / 2}\left(\sum_{n=1}^{r}\left(\frac{\psi\left(Q_{n}\right)}{Q_{n}}\right)^{2}\right)^{1 / 2} .
$$

Similarly, the following theorem also can be established.

Theorem 2.5. Let $P_{i, m_{i}},\left\{a_{i, m_{i}}\right\},\left\{p_{i, m_{i}}\right\}, k_{i}, p$, and $q$ be as in Theorem 2.3 and define $A_{i, m_{i}}=$ $\left(1 / P_{i, m_{i}}\right) \sum_{s_{i}=1}^{m_{i}} p_{i, s_{i}} a_{i, s_{i}}$, for $m_{i}=1,2, \ldots, k_{i}$. Let $\phi_{i}(i=1,2, \ldots, n)$ be $n$ real-valued, nonnegative, and concave functions defined on $R_{+}$.Then,

$$
\sum_{m_{1}=1}^{k_{1}} \cdots \sum_{m_{n}=1}^{k_{n}} \frac{\prod_{i=1}^{n} P_{i, m_{i}} \phi_{i}\left(A_{i, m_{i}}\right)}{\left((1 / n) \sum_{i=1}^{n} m_{i}^{r}\right)^{n /(p r)}} \geq \prod_{i=1}^{n} k_{i}^{1 / p}\left(\sum_{m_{i}=1}^{k_{i}}\left(k_{i}-m_{i}+1\right)\left(p_{i, m_{i}} \phi_{i}\left(a_{i, m_{i}}\right)\right)^{q}\right)^{1 / q} .
$$

The proof of Theorem 2.5 can be completed by following the same steps as in the proof of Theorem 2.3 with suitable changes. Here, we omit the details.

Remark 2.6. Taking $n=2, q=-2, r=-1$ to (2.18), (2.18) becomes

$$
\begin{aligned}
& \sum_{m_{1}=1}^{k_{1}} \sum_{m_{2}=1}^{k_{2}} \frac{P_{1, m_{1}} P_{2, m_{2}} \phi_{1}\left(A_{1, m_{1}}\right) \phi_{2}\left(A_{2, m_{2}}\right)}{\left(m_{1}^{-1}+m_{2}^{-1}\right)^{-3}} \\
& \geq 8\left(k_{1} k_{2}\right)^{3 / 2}\left(\sum_{m_{1}=1}^{k_{1}}\left(k_{1}-m_{1}+1\right)\left(p_{1, m_{1}} \phi_{1}\left(a_{1, m_{1}}\right)\right)^{-2}\right)^{-1 / 2}\left(\sum_{m_{2}=1}^{k_{2}}\left(k_{2}-m_{2}+1\right)\left(p_{2, m_{2}} \phi_{2}\left(a_{2, m_{2}}\right)\right)^{-2}\right)^{-1 / 2} .
\end{aligned}
$$


This is just an inverse of the following inequality which was proven by Pachpatte [9]:

$$
\begin{aligned}
& \sum_{m=1}^{k} \sum_{n=1}^{r} \frac{P_{m} Q_{n} \phi\left(A_{m}\right) \psi\left(B_{n}\right)}{m+n} \\
& \quad \leq \frac{1}{2}(k r)^{1 / 2}\left(\sum_{m=1}^{k}(k-m+1)\left(p_{m} \phi\left(a_{m}\right)\right)^{2}\right)^{1 / 2}\left(\sum_{n=1}^{r}(r-n+1)\left(q_{n} \psi\left(b_{n}\right)\right)^{2}\right)^{1 / 2} .
\end{aligned}
$$

Remark 2.7. In view of L'Hôpital law, we have the following fact:

$$
\begin{aligned}
\lim _{r \rightarrow 0}\left(\frac{1}{n} \sum_{i=1}^{n} m_{i}^{r}\right)^{n /(p r)} & =\exp \left(\frac{n}{p} \lim _{r \rightarrow 0} \frac{\ln \left((1 / n) \sum_{i=1}^{n} m_{i}^{r}\right)}{r}\right) \\
& =\exp \left(\frac{n}{p} \lim _{r \rightarrow 0} \frac{\sum_{i=1}^{n} m_{i}^{r} \ln m_{i}}{\sum_{i=1}^{n} m_{i}^{r}}\right)=\left(m_{1} \cdot m_{2} \cdots m_{n}\right)^{1 / p} .
\end{aligned}
$$

Accordingly, in the special case when $n=2, p=0.1$, and $p_{i, m_{i}}=1$, let $r \rightarrow 0$, then the inequality (2.18) reduces to the following inequality:

$$
\begin{aligned}
\sum_{m_{1}=1}^{k_{1}} & \sum_{m_{2}=1}^{k_{2}} \frac{\phi_{1}\left(A_{1, m_{1}}\right) \phi_{2}\left(A_{2, m_{2}}\right)}{\left(m_{1} m_{2}\right)^{-2}} \\
& \geq\left(k_{1} k_{2}\right)^{-1}\left(\sum_{m_{1}=1}^{k_{1}}\left(k_{1}-m_{1}+1\right)\left(\phi_{1}\left(a_{1, m_{1}}\right)\right)^{1 / 2}\right)^{2}\left(\sum_{m_{2}=1}^{k_{2}}\left(k_{2}-m_{2}+1\right)\left(\phi_{2}\left(a_{2, m_{2}}\right)\right)^{1 / 2}\right)^{2} .
\end{aligned}
$$

This is just a discrete form of the following inequality which was proven by Zhao and Debnath [11]:

$$
\int_{0}^{x} \int_{0}^{y} \frac{\phi(F(s)) \psi(G(t))}{(s t)^{-2}} d s d t \geq(x y)^{-1}\left[\int_{0}^{x}(x-s)\{\phi(f(s))\}^{1 / 2} d s\right]^{2}\left[\int_{0}^{y}(y-t)\{\phi(g(t))\}^{1 / 2} d t\right]^{2} .
$$

\section{Acknowledgments}

The authors cordially thank the anonymous referee for his/her valuable comments which lead to the improvement of this paper. Research is supported by Zhejiang Provincial Natural Science Foundation of China, Grant no. Y605065, Foundation of the Education Department of Zhejiang Province of China, Grant no. 20050392, partially supported by the Research Grants Council of the Hong Kong SAR, China, Project no. HKU7016/07P.

\section{References}

[1] G. D. Handley, J. J. Koliha, and J. E. Pečarić, “New Hilbert-Pachpatte type integral inequalities," Journal of Mathematical Analysis and Applications, vol. 257, no. 1, pp. 238-250, 2001.

[2] G. Minzhe and Y. Bicheng, "On the extended Hilbert's inequality," Proceedings of the American Mathematical Society, vol. 126, no. 3, pp. 751-759, 1998. 
[3] G. Minzhe, "On Hilbert's inequality and its applications," Journal of Mathematical Analysis and Applications, vol. 212, no. 1, pp. 316-323, 1997.

[4] K. Hu, "On Hilbert inequality and its application," Advances in Mathematics, vol. 22, no. 2, pp. 160-163, 1993.

[5] K. Jichang, "On new extensions of Hilbert's integral inequality," Journal of Mathematical Analysis and Applications, vol. 235, no. 2, pp. 608-614, 1999.

[6] Y. Bicheng, "On new generalizations of Hilbert's inequality," Journal of Mathematical Analysis and Applications, vol. 248, no. 1, pp. 29-40, 2000.

[7] C.-J. Zhao, "Inverses of disperse and continuous Pachpatte's inequalities," Acta Mathematica Sinica, vol. 46, no. 6, pp. 1111-1116, 2003.

[8] C.-J. Zhao, "Generalization on two new Hilbert type inequalities," Journal of Mathematics, vol. 20, no. 4, pp. 413-416, 2000.

[9] B. G. Pachpatte, "On some new inequalities similar to Hilbert's inequality," Journal of Mathematical Analysis and Applications, vol. 226, no. 1, pp. 166-179, 1998.

[10] G. H. Hardy, J. E. Littlewood, and G. Pólya, Inequalities, Cambridge University Press, Cambridge, UK, 2nd edition, 1934.

[11] C.-J. Zhao and L. Debnath, "Some new inverse type Hilbert integral inequalities," Journal of Mathematical Analysis and Applications, vol. 262, no. 1, pp. 411-418, 2001. 